\title{
Contamination assessment of Krakow Valleys Landscape Parks surface and wastewaters
}

\author{
Magdalena Strzebońska ${ }^{1}$, Elżbieta Jarosz-Krzemińska ${ }^{2}$, Ewa Adamiec ${ }^{1}$ \\ ${ }^{1}$ AGH University of Science and Technology, Faculty of Geology, Geophysics and Environmental Protection; \\ al.Mickiewicza 30,30-059 Krakow,Poland; e-mail:mstrzebo@agh.edu.pl,eadamiec@agh.edu.pl \\ ${ }^{2}$ AGH University of Science and Technology; al. Mickiewicza 30, 30-059 Krakow, Poland; \\ e-mail: elzbietajarosz@geol.agh.edu.pl (correspondingauthor)
}

(C) 2014 Authors. This is an open access publication, which can be used, distributed and reproduced in any medium according to the Creative Commons CC-BY 4.0 License requiring that the original work has been properly cited.

Received: 18 December 2014; accepted: 6 February 2015

\begin{abstract}
This research consisted of a physico-chemical evaluation of the streams and wastewaters within Krakow Valleys Landscape Park in the Kluczwody, Bolechowicka, Będkowska and Kobylańska valleys. The following set of parameters were determined in the samples: $\mathrm{pH}$, Eh, conductivity, oxygen content, concentration of metals' cations and selected anions. The results revealed that the wastewaters discharged as well as the streams that feed into the Rudawa river are mainly polluted with nitrates. Additionally, the examined wastewaters contained a high concentration of phosphate exceeding class II purity maximum permissible values.
\end{abstract}

Keywords: contamination, stream, wastewater, Krakow Valleys Landscape Park

\section{INTRODUCTION}

Poland's surface waters are considered to be the most polluted component in its environment, where water pollution is regarded as physico-chemical or biological changes which lower the quality of waters (Bielak 2014). Landscape parks are expressly created forms of nature conservation. In Polish law, special care with regards to soil, water and air pollution, is given to protected areas such as landscape parks. Yet it is worth mentioning that this type of protected natural area in Poland has a lower status than national parks and is subject to less stringent restrictions. However, Krakow Valleys Landscape Park, like all other landscape parks in Poland, is protected by law under Voivodeship Regulatory Act no. 82/06 from October $17^{\text {th }}$ of 2006 (Rozporzadzenie Nr 82/06 Wojewody Małopolskiego...).
The regulation prohibits the following:

- any contamination of water or soil and the use of chemicals within the park,

- any investment projects that may have a significant impact on the environment.

Furthermore, the regulation stipulates that any villages adjacent to the park are obligated to organize proper waste management systems. Additional restrictions, which are included in the local development plans, impose the collective sewage system or the installation of leakproof septic tanks as the only acceptable forms of wastewater management. In the Zabierzów commune the local development plan prioritizes environmental protection objectives placing an emphasis on restoring and sustaining the purity and quality of water (Grzejdziak \& Korzeniak 2010).

Despite the explicit prohibitions and guidelines regarding protection of the environment within 
Krakow Valleys Landscape Park the degradation of water quality, because of domestic wastewater or due to leakage of septic tanks which are often discharged directly into the water bodies and soil, is still a big concern (Waszkiewicz 2005).

The following is a list of the main sources of pollution, with heavy metals and other toxic compounds, in the Krakow Valleys (Kyzioł-Komosińska \& Kukułka 2008, Grzejdziak \& Korzeniak 2010):

- particulate emissions from local boilers and individual furnaces;

- industrial emission from the outside of the study area (province of Silesia, Skawina and Krakow);

- ground transportation pollution, occurring mainly in the vicinity of the national road No. 79 Krakow - Katowice, including A4 motorway Katowice - Krakow;

- uncontrolled wastewater run-off from households;

- production and use of fertilizers and pesticides in horticulture and agriculture.
The main objective of the study was to assess the degree of contamination of the selected streams and wastewater discharged directly to the Rudawa river in the Krakow Valleys Landscape Park.

\section{STUDY AREA}

Krakow Valleys Landscape Park (Fig. 1) belongs to the Jurassic Landscape Park and is located in the Southern part of the Krakow Upland. It comprises of left-bank tributaries of the Rudawa River, upper part of the Sztoła River along with the forests on South of Bukowno and lower course of the Prądnik River. The park covers an area of approximately 20,686 hectares, and is located partially within the Bukowno, Jerzmanowice-Przeginia, Krakow, Krzeszowice, Michałowice, Olkusz, Trzebinia, Wielka Wieś, Zabierzów and Zielonki communes (Uchwała nr XV/247/11 Sejmiku Województwa Małopolskiego...).

The study area is located within the Silesian-Cracow Upland and almost entirely belongs to the subdivision of the Krakowsko-Częstochowska Upland, the part of the Olkuska Upland (Kondracki 2000).

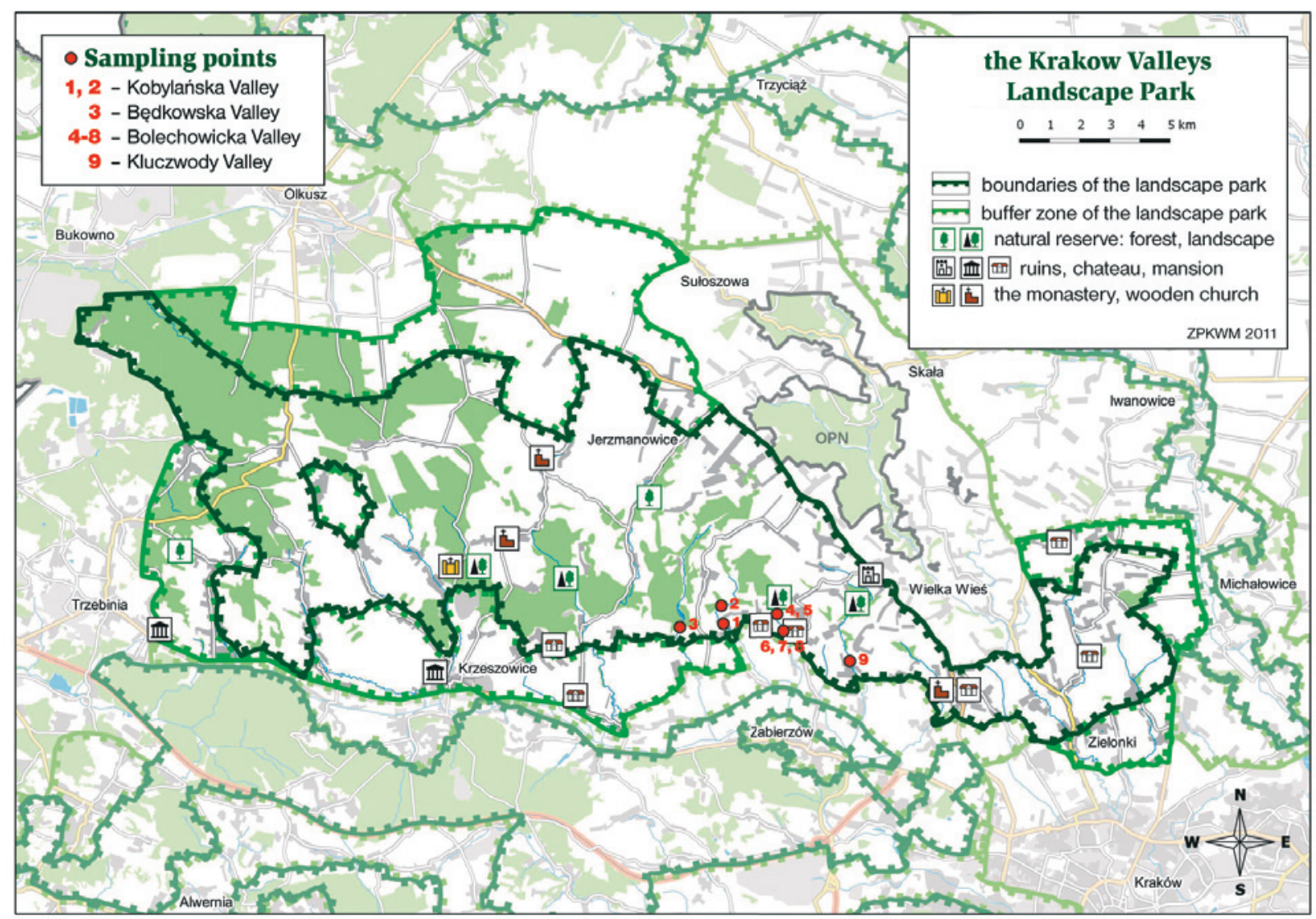

Fig. 1. Study area and sampling points (based on ZPKWM map; www.zpkwm.pl/krakow/parki.html) 
The Olkuska Upland is a plateau consisting of carbonate rocks of Triassic and Upper Jurassic. These rocks have a corrugated surface, cut off from the southern site by a steep straightforward (local declines up to $70 \%$ ) edge which separates upland from the Krzeszowice Trench. The Kobylańska and Bolechowicka valleys are cut by deep valleys and gorges (Pasieczna 2012), slopes of which are diversified with numerous limestone rocks.

\section{SAMPLING}

Water samples were collected in March 2014 from the Rudawa’s River tributaries: Będkówka, Kobylanka, Bolechówka and Kluczwoda streams and from the wastewaters running off directly to these streams. Sampling points are presented on Figure 1.

The streams under investigation were direct left-bank tributaries of the Rudawa River (Kobylanka, Będkówka, Kluczwoda) as well as streams flowing into the Krzeszowice Trench at the Kobylanka (Bolechówka) stream juncture.

\section{METHODS}

Geochemical evaluation was performed on streams' water as well as on wastewaters. Physico-chemical parameters such as conductivity, $\mathrm{pH}$ and oxygen content were measured in situ using Thermo Scientific Orion Star A329 Portable Multiparameter Meter. Water samples were filtered prior analysis through $0.45 \mu \mathrm{m}$ Millipore filter. Concentrations of the following cations: $\mathrm{Ag}, \mathrm{Al}$, As, B, Ba, Ca, Cd, Co, Cr, Cu, Fe, Hg, K, Li, Mg, $\mathrm{Mn}, \mathrm{Mo}, \mathrm{Na}, \mathrm{Ni}, \mathrm{Pb}, \mathrm{Sb}, \mathrm{Si}, \mathrm{Sn}, \mathrm{Sr}, \mathrm{Zn}$ were analyzed using ICP-OES method. Nitrate nitrogen (V) was determined in the water samples using spectrophotometric method with sodium salicylate according to $\mathrm{PN}-82 / \mathrm{C}-04576.08$ and chlorides using titration method with silver nitrate in the presence of chromate as indicator (Mohr's method) according to ISO 9297:1994. Phosphates were ascertained using ammonium molybdate spectrophotometry method according to PN-EN ISO 6878: 2006/Ap2:2010.

\section{RESULTS AND DISCUSSION}

The obtained results when compared with the threshold values of the water quality indicators according to Polish Directive - Journal of Laws 2011 no. 257, item 1545 (Rozporzadzenie Ministra Środowiska...) revealed that the water from the streams meet class II purity requirements (due to high concentration of nitrate nitrogen and calcium). All examined streams and wastewaters meet I purity class with regards to physical parameters such as conductivity (below $1000 \mu \mathrm{S} / \mathrm{cm}$ ) and dissolved oxygen ( $\geq 7 \mathrm{mg} / \mathrm{L}$ ). Conductivity changes from $228 \mu \mathrm{S} / \mathrm{cm}$ to $856 \mu \mathrm{S} / \mathrm{cm}$ and dissolved oxygen varies from $9.2 \mathrm{mg} / \mathrm{L}$ to $12.6 \mathrm{mg} / \mathrm{L}$. However, the concentration of calcium is of natural origin resulting from the carbonate rocks. The results of analysis for the wastewater discharged into Bolechówka and Kobylanka streams revealed a high concentration of phosphate exceeding the threshold values for the class II purity rating. In addition, the wastewater running off into the Bolechówka stream revealed high $\mathrm{pH}$, which indicate the quality of the stream below acceptable (Tab. 1). Both streams and wastewater exceeded the limits for I purity class with regards to arsenic (wastewater flowing into Kobylanka stream), antimony, selenium and vanadium. Moreover in the Będkówka stream the concentration of mercury exceeded the maximum threshold values for water quality according to the legislation (Tab. 2). The obtained research results are in agreement with the 2013 assessment study of the Regional Inspectorate of Environmental Protection in Krakow (WIOŚ) carried out at the Rudawa-Krakow control point. This evaluation considered surface water from the Racławka stream up to the estuary of the Rudawa into Vistula River. The results revealed an increased content of nitrate nitrogen $(3.17 \mathrm{mg} / \mathrm{L})$. Surface water contaminated with nitrate affects groundwater contamination in this area. The collected results of this study are therefore convergent with those of Żurek et al. (2010), indicating that nitrates are the main contaminant of groundwater in this region.

In order to fully assess the degree of water pollution in Krakow Valleys Landscape Park, research findings regarding physico-chemical parameters of the waterbodies should be supplemented by the biological indicators results obtained from the WIOS monitoring data. The combination of physico-chemical evaluation with bioindication analysis is the most comprehensive approach in determining water quality (Krzeminska 2004, Sadowska 2012). 


\begin{tabular}{|c|c|c|c|c|c|c|c|c|c|c|c|c|c|c|c|c|}
\hline \multirow{2}{*}{ 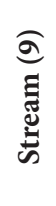 } & \multirow{2}{*}{ 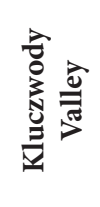 } & $\begin{array}{l}\frac{0}{0} \\
\frac{\tilde{\sigma}}{0}\end{array}$ & $\neg$ & - & $\neg$ & $\curvearrowleft$ & $\neg$ & $\Xi$ & - & $=$ & I & $=$ & 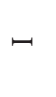 & I & I & \\
\hline & & $\frac{\mathscr{\Xi}}{\tilde{J}}$ & 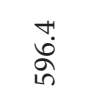 & $\begin{array}{l}\infty \\
\infty \\
\stackrel{0}{\hookrightarrow}\end{array}$ & $\underset{\infty}{\stackrel{\infty}{a}}$ & $\begin{array}{l}\stackrel{\infty}{\infty} \\
\stackrel{a}{\beth}\end{array}$ & $\begin{array}{l}\text { ஜे } \\
\text { : }\end{array}$ & $\underset{\sim}{\stackrel{H}{m}}$ & $\frac{\text { fे }}{\stackrel{m}{-}}$ & $\begin{array}{l}8 \\
\text { ì } \\
\text { d. }\end{array}$ & $\begin{array}{l}\Delta \\
\stackrel{0}{0}\end{array}$ & کָּ & $\begin{array}{l}\stackrel{゚}{2} \\
i n\end{array}$ & 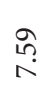 & 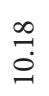 & \\
\hline \multirow{2}{*}{ 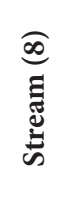 } & \multirow{2}{*}{ 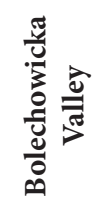 } & $\begin{array}{l}\text { D } \\
\frac{\pi}{0}\end{array}$ & $\neg$ & $\neg$ & 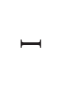 & 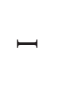 & 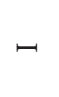 & $\neg$ & $\neg$ & $\Xi$ & $=$ & $=$ & $\neg$ & $=$ & $=$ & \\
\hline & & $\frac{\mathscr{y}}{\frac{\Xi}{5}}$ & $\begin{array}{l}\infty \\
\text { ळ். } \\
\sigma\end{array}$ & $\begin{array}{l}\stackrel{\text { +े }}{\circ} \\
\stackrel{-}{2}\end{array}$ & $\stackrel{\infty}{\stackrel{\infty}{\wedge}}$ & $\stackrel{\sim}{m}$ & $\begin{array}{l}\ddot{n} \\
\stackrel{0}{0}\end{array}$ & $\stackrel{\infty}{\stackrel{\infty}{-}}$ & $\begin{array}{l}\vec{\Xi} \\
\dot{\mathrm{i}}\end{array}$ & $\begin{array}{l}\text { H. } \\
\text { ò }\end{array}$ & $\hat{o}$ & $\stackrel{\sim}{\sim}$ & $\begin{array}{l}\infty \\
\stackrel{-}{\rightarrow}\end{array}$ & $\begin{array}{l}\qquad 0 \\
0 \\
0\end{array}$ & 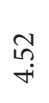 & \\
\hline \multirow{2}{*}{ 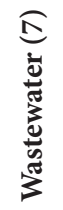 } & \multirow{2}{*}{ 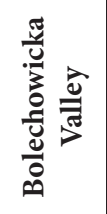 } & $\begin{array}{l}\frac{0}{0} \\
\frac{\pi}{0}\end{array}$ & $\neg$ & - & $\neg$ & - & @i & $\Xi$ & - & $\Xi$ & $=$ & $=$ & $\neg$ & $=$ & $=$ & \\
\hline & & $\frac{\cong}{\tilde{J}}$ & $\begin{array}{l}n \\
\varnothing \\
\infty \\
\infty\end{array}$ & 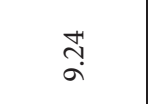 & $\stackrel{\infty}{\stackrel{\infty}{\sim}}$ & $\begin{array}{l}\hat{\pi} \\
\infty \\
\text { n}\end{array}$ & ڤે & $\begin{array}{l}\infty \\
\infty \\
\end{array}$ & $\begin{array}{l}\stackrel{N}{\hat{m}} \\
\stackrel{\bar{m}}{2}\end{array}$ & $\begin{array}{l}\stackrel{\sim}{ } \\
\infty \\
\stackrel{\sim}{\sim}\end{array}$ & $\begin{array}{l}\text { Oे } \\
\stackrel{\circ}{0}\end{array}$ & $\stackrel{\vec{n}}{=}$ & $\stackrel{+}{\mathrm{I}}$ & $\stackrel{m}{a}$ & 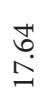 & \\
\hline \multirow{2}{*}{ 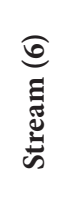 } & \multirow{2}{*}{ 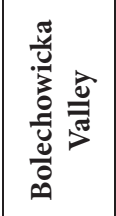 } & $\begin{array}{l}\text { 总 } \\
\frac{\pi}{0}\end{array}$ & 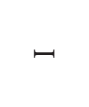 & $\neg$ & $\neg$ & $\neg$ & 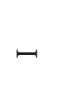 & $=$ & $\neg$ & $\neg$ & $=$ & $=$ & $\neg$ & $=$ & s & \\
\hline & & $\frac{\mathscr{E}}{\frac{E}{5}}$ & $\begin{array}{c}m \\
\stackrel{H}{+}\end{array}$ & 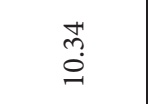 & $\underset{\infty}{\stackrel{?}{\infty}}$ & $\stackrel{\alpha}{\alpha}$ & ஜ̊ & $\underset{\sim}{\stackrel{H}{~}}$ & $\begin{array}{l}\stackrel{0}{ } \\
\stackrel{I}{I}\end{array}$ & $\begin{array}{l}\infty \\
\infty \\
\stackrel{R}{ }\end{array}$ & $\tilde{o}$ & חֶ? & $\underset{\vec{n}}{i}$ & $\stackrel{\infty}{\stackrel{\infty}{\infty}}$ & $\stackrel{m}{\sigma}$ & \\
\hline \multirow{2}{*}{ 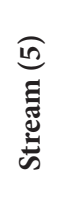 } & \multirow{2}{*}{ 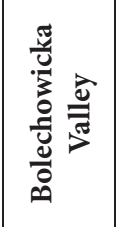 } & 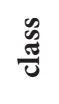 & $\neg$ & - & $\neg$ & $\neg$ & $\neg$ & $\Xi$ & $\neg$ & $\neg$ & $=$ & $=$ & $\neg$ & $=$ & s & \\
\hline & & $\frac{\mathscr{\Xi}}{\mathrm{E}}$ & 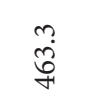 & $\begin{array}{l}\infty \\
\infty \\
a\end{array}$ & $\stackrel{\vec{\infty}}{\infty}$ & $\stackrel{\oplus}{=}$ & $\begin{array}{l}\mathscr{2} \\
\stackrel{2}{0} \\
0\end{array}$ & $\underset{\dot{m}}{\vec{n}}$ & $\begin{array}{l}0 \\
\stackrel{1}{\beth}\end{array}$ & $\begin{array}{l}\text { 오 } \\
\stackrel{0}{\circ}\end{array}$ & $\stackrel{\infty}{0}$ & aे & $\begin{array}{l}\mathscr{\infty} \\
\stackrel{\sim}{*}\end{array}$ & $\stackrel{\stackrel{?}{+}}{+}$ & $\begin{array}{l}\text { } \\
\stackrel{\varrho}{0}\end{array}$ & \\
\hline \multirow{2}{*}{ 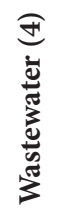 } & \multirow{2}{*}{ 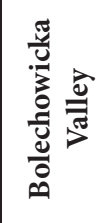 } & $\begin{array}{l}\text { 足 } \\
\text { 吾 }\end{array}$ & - & $\neg$ & 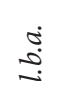 & - & $\neg$ & $\neg$ & $\neg$ & $\neg$ & $=$ & $=$ & $\neg$ & I & $=$ & \\
\hline & & $\frac{\mathscr{E}}{\mathrm{E}}$ & $\begin{array}{l}\stackrel{+}{\infty} \\
\stackrel{\sim}{\sim} \\
\text {. }\end{array}$ & $\begin{array}{l}\tilde{\sigma} \\
\stackrel{\bigcup}{J}\end{array}$ & $\stackrel{H}{a}$ & $\stackrel{\stackrel{m}{=}}{=}$ & $\stackrel{1}{0}$ & $\stackrel{\text { I }}{-}$ & $\begin{array}{l}0 \\
\text { mn } \\
\stackrel{n}{n}\end{array}$ & $\frac{\tilde{o}}{\dot{m}}$ & તู่ & $\stackrel{\text { f }}{\text { i }}$ & $\stackrel{\Omega}{\text { }}$ & $\stackrel{\vec{S}}{\mathrm{i}}$ & $\stackrel{\infty}{\stackrel{\infty}{\wedge}}$ & \\
\hline \multirow{2}{*}{ 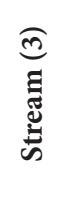 } & \multirow{2}{*}{ 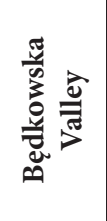 } & 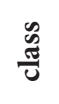 & - & - & - & $\neg$ & $\neg$ & $\exists$ & - & $\neg$ & $=$ & $=$ & $\neg$ & $=$ & s & \\
\hline & & $\frac{\mathscr{y}}{\frac{\Xi}{J}}$ & 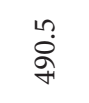 & $\begin{array}{l}\infty \\
\infty \\
\stackrel{0}{-}\end{array}$ & $\underset{\infty}{\mathbb{\infty}}$ & $\begin{array}{l}\stackrel{゚}{ } \\
\stackrel{\text { I }}{ }\end{array}$ & त्ठ & $\underset{m}{\vec{n}}$ & 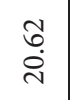 & $\begin{array}{l}\text { ڤn } \\
\text { Lू. }\end{array}$ & $\overrightarrow{\tilde{m}}$ & $\underset{F}{F}$ & $\stackrel{H}{i}$ & $\stackrel{\infty}{\stackrel{\infty}{H}}$ & $\begin{array}{c}\infty \\
\stackrel{\infty}{\infty} \\
\end{array}$ & \\
\hline \multirow{2}{*}{ 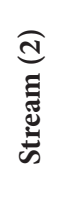 } & \multirow{2}{*}{ 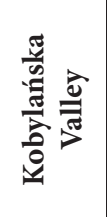 } & 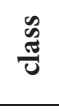 & - & - & $\neg$ & - & $\neg$ & $\Xi$ & $\neg$ & $\exists$ & $=$ & $=$ & $\neg$ & $=$ & $=$ & \\
\hline & & $\frac{\mathscr{E}}{\tilde{J}}$ & $\begin{array}{l}\text { Oे. } \\
\text { in }\end{array}$ & $\begin{array}{l}0 \\
\stackrel{1}{0} \\
0\end{array}$ & $\overrightarrow{0}$ & & $\begin{array}{l}\text { İ } \\
\text { : }\end{array}$ & $\begin{array}{l}\stackrel{n}{n} \\
n \\
n\end{array}$ & 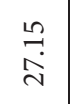 & $\begin{array}{l}\stackrel{\partial}{+} \\
\stackrel{+}{=}\end{array}$ & $\underset{\text { ö }}{\text {. }}$ & ڤิ & $\begin{array}{l}\text { है } \\
\text { in }\end{array}$ & $\stackrel{\text { gे }}{+}$ & $\stackrel{\overbrace{}}{=}$ & \\
\hline \multirow{2}{*}{ 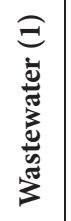 } & \multirow{2}{*}{ 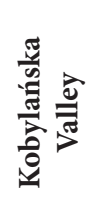 } & $\begin{array}{l}\text { D } \\
\frac{\tilde{J}}{\mathrm{~d}}\end{array}$ & $\neg$ & - & - & - & : & $\neg$ & $\neg$ & $\exists$ & $=$ & $=$ & - & I & $=$ & \\
\hline & & $\frac{\mathscr{E}}{\tilde{E}}$ & $\underset{\mathbb{C}}{\vec{f}}$ & $\stackrel{\circ}{\stackrel{0}{0}}$ & $\stackrel{m}{n}$ & $\stackrel{\partial}{\circ}$ & $\underset{\hat{n}}{N}$ & $\stackrel{+m}{-}$ & $\frac{\stackrel{f}{m}}{\dot{m}}$ & $\begin{array}{l}\stackrel{m}{\leftrightarrow} \\
\stackrel{0}{\leftrightarrows}\end{array}$ & $\stackrel{\Rightarrow}{m}$ & 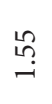 & $\stackrel{\hat{n}}{=}$ & $\begin{array}{l}\stackrel{0}{0} \\
\infty\end{array}$ & $\begin{array}{l}\text { İ } \\
\text { İ }\end{array}$ & \\
\hline \multicolumn{3}{|c|}{ 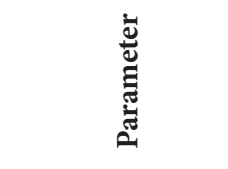 } & 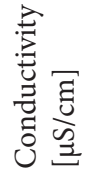 & 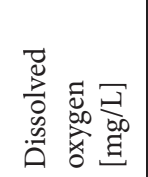 & 兑 & $\begin{array}{l}\frac{\vec{\sigma}}{\Delta, 0} \\
\frac{\Xi}{\sigma}\end{array}$ & 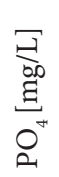 & 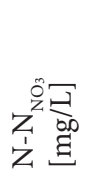 & $\begin{array}{l}\vec{F} \\
\stackrel{50}{\Xi} \\
\dot{D}^{+}\end{array}$ & 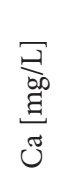 & 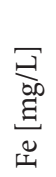 & 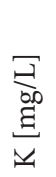 & 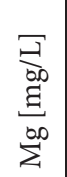 & 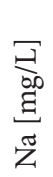 & $\begin{array}{l}\vec{F} \\
\vec{b} \\
\dot{\omega}\end{array}$ & 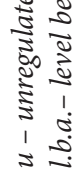 \\
\hline
\end{tabular}




\begin{tabular}{|c|c|c|c|c|c|c|c|c|c|c|c|c|c|c|c|c|c|c|c|c|c|}
\hline 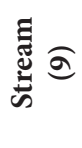 & 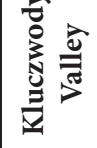 & & $\stackrel{\vec{\partial}}{\vec{v}}$ & $\stackrel{m}{m}$ & 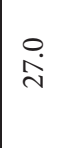 & 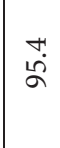 & 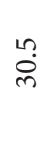 & $\vec{\partial}$ & $\stackrel{\sim}{\rightarrow}$ & $\stackrel{0}{0}$ & $\stackrel{a}{\infty}$ & $\overrightarrow{\vec{d}}$ & $\stackrel{\sharp}{-}$ & $\begin{array}{l}\vec{b} \\
\dot{n}\end{array}$ & $\stackrel{i}{i}$ & $\underset{v}{\vec{v}}$ & $\vec{\sigma}$ & $\overrightarrow{\vec{\sigma}}$ & $\stackrel{\vec{g}}{\vec{g}}$ & $\stackrel{\leftrightarrow}{N}$ & \\
\hline 志 & 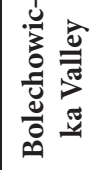 & & $\vec{d}$ & $F$ & 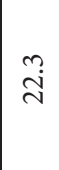 & $\begin{array}{l}\vec{H} \\
\stackrel{0}{\sigma}\end{array}$ & ָू & $\vec{\partial}$ & $\stackrel{\infty}{-}$ & $\ddot{0}$ & ${ }_{\infty}^{0}$ & $\underset{v}{\vec{v}}$ & $\hat{i}$ & $\underset{\infty}{\infty}$ & ?ִ & $\underset{v}{\vec{v}}$ & $\vec{\sigma}$ & $\overrightarrow{\vec{\sigma}}$ & $\underset{\sim}{\sim}$ & $\begin{array}{l}0 \\
\ddot{g}\end{array}$ & \\
\hline 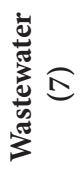 & 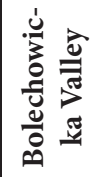 & & $\vec{d}$ & $\vec{d}$ & $\vec{a}$ & $\stackrel{g}{\underline{\Delta}}$ & $\begin{array}{l}\text { In } \\
\text { in }\end{array}$ & $\vec{d}$ & $\stackrel{m}{i}$ & $\stackrel{1}{0}$ & $\stackrel{+}{0}$ & $\vec{d}$ & $\stackrel{H}{m}$ & $\stackrel{\sim}{\sim}$ & $\stackrel{\infty}{i}$ & $\vec{\nabla}$ & $\vec{\nabla}$ & $\vec{\sigma}$ & 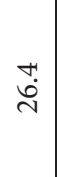 & $\begin{array}{l}\text { ம் } \\
\stackrel{\sim}{ }\end{array}$ & \\
\hline 氖 & 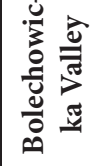 & & $\underset{i}{F}$ & $\stackrel{\infty}{\rightarrow}$ & $\stackrel{\sim}{\stackrel{m}{m}}$ & $\begin{array}{l}0 \\
\dot{0} \\
0\end{array}$ & 吕 & $\vec{\partial}$ & $\tilde{i}$ & $\stackrel{\circ}{\rightarrow}$ & $\vec{a}$ & $\underset{v}{\vec{d}}$ & $\stackrel{4}{0}$ & $\stackrel{m}{-}$ & $\stackrel{\sim}{i}$ & $\overrightarrow{\vec{d}}$ & $\vec{\partial}$ & $\stackrel{+}{0}$ & $\underset{\infty}{\infty}$ & $\begin{array}{l}\infty \\
\dot{\varphi} \\
0\end{array}$ & \\
\hline 馬 & 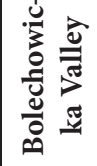 & $\underset{\Xi}{\overrightarrow{00}}$ & $\vec{v}$ & $\stackrel{\infty}{i}$ & $\stackrel{\rho}{\infty}$ & $\hat{\hat{\sigma}}$ & $\overrightarrow{\vec{N}}$ & $\stackrel{m}{0}$ & $\vec{d}$ & $\stackrel{\circ}{\rightarrow}$ & $\vec{\partial}$ & $\overrightarrow{\vec{d}}$ & $\stackrel{0}{\circ}$ & $\stackrel{\circ}{i}$ & iี & $\overrightarrow{\mathrm{d}}$ & $\overrightarrow{\mathrm{O}}$ & $\stackrel{0}{i}$ & $\stackrel{\circ}{\exists}$ & $\stackrel{\infty}{\wedge}$ & \\
\hline 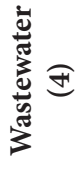 & 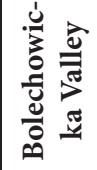 & & $\vec{\partial}$ & $\stackrel{\circ}{\underset{+}{+}}$ & & $\begin{array}{l}4 \\
0 \\
0\end{array}$ & 富 & $\vec{d}$ & $\overrightarrow{\vec{d}}$ & $\hat{o}$ & $\overrightarrow{\mathrm{i}}$ & $\underset{v}{\vec{\sigma}}$ & $\stackrel{H}{0}$ & $\overrightarrow{6}$ & 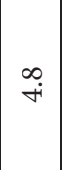 & $\vec{d}$ & $\vec{\nabla}$ & $\widehat{\sim}$ & $\stackrel{\beth}{\exists}$ & ลे & \\
\hline 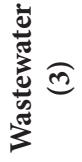 & 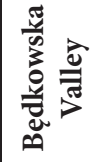 & & $\vec{\sigma}$ & $\stackrel{\sim}{\sim}$ & $\stackrel{\stackrel{n}{\lambda}}{\vec{v}}$ & $\stackrel{\circ}{=}$ & $\stackrel{\circ}{\infty}$ & $\vec{\nabla}$ & $\overrightarrow{\vec{\sigma}}$ & $\stackrel{\infty}{\circ}$ & $\stackrel{n}{\longrightarrow}$ & $\stackrel{m}{-}$ & $\hat{0}$ & $\stackrel{?}{=}$ & $\stackrel{n}{m}$ & $\overrightarrow{\vec{v}}$ & $\overrightarrow{\mathrm{\sigma}}$ & $\stackrel{m}{\varrho}$ & 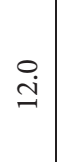 & 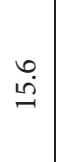 & \\
\hline 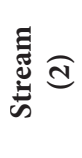 & 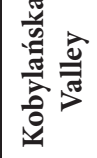 & & $\vec{\partial}$ & $\stackrel{\dot{m}}{ }$ & ֻै & $\stackrel{+}{\infty}$ & ஷุ & $\vec{\sigma}$ & $\vec{\partial}$ & $\stackrel{0}{-}$ & $\vec{\partial}$ & $\underset{v}{\vec{O}}$ & $\stackrel{\circ}{\rightarrow}$ & $\stackrel{n}{\longrightarrow}$ & $\stackrel{\circ}{i}$ & $\overrightarrow{\vec{v}}$ & $\vec{\partial}$ & $\stackrel{20}{2}$ & $\stackrel{\vec{\vartheta}}{\vec{g}}$ & $\vec{I}$ & \\
\hline 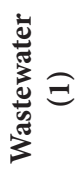 & 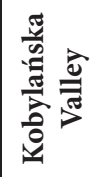 & & $\vec{\partial}$ & $\begin{array}{l}\infty \\
\stackrel{i}{i}\end{array}$ & $\stackrel{+}{0}$ & $\stackrel{m}{\stackrel{n}{N}}$ & $\frac{N}{m}$ & $\hat{\circ}$ & $\vec{\nabla}$ & ò & iे & $\vec{\partial}$ & $\vec{i}$ & $\exists$ & 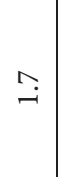 & $\overrightarrow{\vec{v}}$ & $\vec{\nabla}$ & $\stackrel{\infty}{\stackrel{i}{i}}$ & $\overrightarrow{\stackrel{g}{n}}$ & نْ & 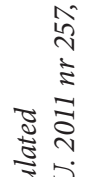 \\
\hline $\begin{array}{l}\overline{7} \\
\overline{0} \\
\bar{g} \\
\bar{g} \\
\bar{E}\end{array}$ & 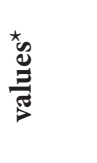 & $\underset{0}{\stackrel{1}{00}}$ & ir & $\begin{array}{l}\text { 就 } \\
\text { a }\end{array}$ & in & $\begin{array}{l}\text { ¿ें } \\
\text { ¿ें }\end{array}$ & $\begin{array}{l}\text { Pें } \\
\text { ¿ें }\end{array}$ & $\stackrel{n}{\longrightarrow}$ & in & $\begin{array}{l}0 \\
\text { in }\end{array}$ & $\begin{array}{l}\text {. } \\
\text { in }\end{array}$ & $\stackrel{\text { Oạ }}{0}$ & 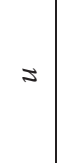 & $=$ & $\begin{array}{l}\text {. } \\
\stackrel{+}{+}\end{array}$ & 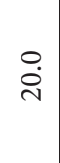 & $\stackrel{?}{\wedge}$ & 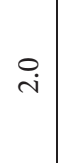 & $=$ & $\begin{array}{l}\stackrel{0}{\dot{0}} \\
\stackrel{0}{0}\end{array}$ & 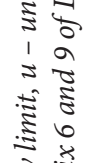 \\
\hline & 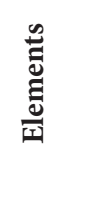 & & ${ }^{20}$ & 飞 & ${ }^{2}$ & $\infty$ & $\cong$ & $\bar{U}$ & ن & பे & $\Xi$ & $\stackrel{\infty}{\stackrel{1}{\pi}}$ & $\vec{\exists}$ & $\sum_{\Sigma}^{\Xi}$ & $\stackrel{0}{\Sigma}$ & $\bar{z}$ & $\overrightarrow{2}$ & क & מี & ㄱ & 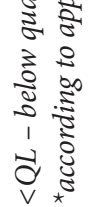 \\
\hline
\end{tabular}


The Rudawa River assessment conducted by WIOS in 2006, at the Podkamycze control point revealed that the river exceeds threshold values with regards to eutrophicationindicators (according to Polish Directive - Journal of Laws 2002 no. 241, item. 2093). One of the highest nitrate concentrations of agricultural origin was detected at this control point. Evaluation of the Rudawa River with respect to other indicators revealed that five-day biochemical oxygen demand $\left(\mathrm{BOD}_{5}\right)$ and phosphates content categorizes this river as class IV purity. With respect to color, Kjeldahl nitrogen, nitrates, nitrites, total nitrogen, manganese, iron concentration the Rudawa River meet class III purity rating. Bacteriological and biological evaluation revealed that the Rudawa River meet class IV purity requirements according to the total number of coliforms and class III purity with respect to fecal coliform bacteria as well as saprobity periphyton index.

\section{CONCLUSIONS}

1. The research results revealed no significant $\mathrm{Zn}$, $\mathrm{Pb}, \mathrm{Cd}$ or $\mathrm{Hg}$ pollution of the streams and wastewater. Concentrations of these heavy metals varied insignificantly within sampling points. The streams under analysis can mostly meet class I purity rating according to the legislation.

2. Despite the fact that the study area is protected by law and therefore the water and wastewater system within its boundaries should be under strict control, there are still many contamination point-sources such as domestic wastewater containing significant loads of $\mathrm{PO}_{4}^{2-}$ and $\mathrm{Cl}^{-}$ anions.

3. Elevated nitrate concentrations in the surface waters within Krakow Valleys Landscape Park could be a consequence of household sewage run-off from nearby infrastructure lacking proper sewerage.

\section{REFERENCES}

Bielak S.R., 2014. Zastosowanie modelowania matematycznego $\mathrm{w}$ analizie zmian stężeń tlenu oraz zakwaszenia wód rzecznych. Aura, 4/2014, 7-10.

Grzejdziak A. \& Korzeniak J., 2010. Prognoza oddziaływania na środowisko ustaleń zmiany studium uwarunkowań i kierunków zagospodarowania przestrzennego gminy
Zabierzów. [on-line:] www.planowanie.zabierzow.org. pl/assets/files/prognoza_oddzialywania_na_srodowisko_studium.pdf [access: September 2014].

Kondracki J., 2000. Geografia regionalna Polski. Wyd. Nauk. PWN, Warszawa.

Krzemińska A., 2004. Testy ekotoksykologiczne, czyli nowe trendy w monitoringu jakości wód powierzchniowych i podziemnych. Nadgorliwość, czy konieczność? Gospodarka Wodna, 1, 19-23.

Kyzioł-Komosińska J. \& Kukułka L., 2008. Wykorzystanie kopalin towarzyszących pokładom złóż węgli brunatnych do usuwania metali ciężkich $z$ wód i ścieków. Series: Works \& Studies. Prace i Studia, 75, Instytut Podstaw Inżynierii Środowiska Polskiej Akademii Nauk, Zabrze.

Pasieczna A., 2012. Geochemiczny zapis skażenia środowiska w rejonie historycznej eksploatacji rud $\mathrm{Zn}-\mathrm{Pb}$ w okolicach Nowej Góry k. Krzeszowic. Biuletyn Państwowego Instytutu Geologicznego, 448 (2), 381-391.

PN-82/C-04576.08: Woda i ścieki - Badania zawartości związków azotu - Oznaczanie azotu azotanowego metoda kolorymetryczna $z$ salicylanem sodowym [Water and waste water tests for nitrogen - Determination of nitrate nitrogen by calorimetric method with sodium salicylate].

PN-ISO 9297:1994: Jakość wody - Oznaczanie chlorków Metoda miareczkowania azotanem srebra $w$ obecności chromianu jako wskaźnika (Metoda Mohra) [Water quality - Determination of chloride - silver nitrate titration with chromate indicator (Mohr's method)].

PN-EN ISO 6878:2006/Ap2:2010: Jakość wody - Oznaczanie fosforu - Metoda spektrometryczna $z$ molibdenianem amonu [Water quality - Determination of phosphorus Spectrometric method with ammonium molybdate].

Rozporządzenie Ministra Środowiska z dnia 23 grudnia 2002 r. w sprawie kryteriów wyznaczania wód wrażliwych na zanieczyszczenie zwiąkami azotu ze źródeł rolniczych. Dz.U. 2002 nr 241 poz. 2093.

Rozporządzenie Ministra Środowiska $z$ dnia 9 listopada $2011 r$. w sprawie sposobu klasyfikacji stanu jednolitych części wód powierzchniowych oraz środowiskowych norm jakości dla substancji priorytetowych. Dz.U. 2011 nr 257 poz. 1545.

Rozporzadzenie Nr 82/06 Wojewody Małopolskiego z dnia 17 października 2006 r. w sprawie Parku Krajobrazowego Dolinki Krakowskie.

Sadowska U., 2012. Ranga bioindykacji w ekotoksykologii wód. Studia Ecologiae et Bioethicae, 10 (2), 33-52.

Uchwała nr XV/247/11 Sejmiku Województwa Małopolskiego $z$ dnia 28 listopada 2011 r. w sprawie Parku Krajobrazowego Dolinki Krakowskie.

Waszkiewicz M., 2005. Jura Krakowska - przyroda i jej zagrożenia. Dzikie Życie, 4 (130). [on-line:] www.pracownia.org.pl/dzikie-zycie-numery-archiwalne, 2126, article,2619 [access: September 2014].

Zespół Parków Krajobrazowych Województwa Małopolskiego (ZPKWM). [on-line:] www.zpkwm.pl/krakow/ parki.html [access: 10.12.2014].

Żurek A., Czop M. \& Motyka J., 2010. Azotany w wodach jurajskiego piętra wodonośnego w rejonie Olkusza. Geologia. Kwartalnik Akademii Górniczo-Hutniczej im. Stanisława Staszica w Krakowie, 36 (1), 109-134. 